\title{
Optical recording from respiratory pattern generator of foetal mouse brainstem reveals a distributed network
}

\author{
Jaime Eugenin ${ }^{1,4}$, John G. Nicholls ${ }^{2,4}$, Lawrence B. Cohen ${ }^{3,4}$ and Kenneth J. Muller ${ }^{5}$ \\ ${ }^{1}$ Dept. of Biology, USACH, Santiago, Chile \\ 2 Sissa, Trieste, Italy \\ ${ }^{3}$ Dept. of Physiology, Yale University School of Medicine, New Haven, CT 06520 \\ ${ }^{4}$ Marine Biological Laboratory, Woods Hole, MA 02543 USA \\ ${ }^{5}$ Dept of Physiology \& Biophysics and Neuroscience Program, University of Miami \\ School of Medicine, Miami, FL 33134 USA
}

Section Editor-Developmental: Dr. John L. R. Rubenstein

Corresponding author: Kenneth J. Muller, Dept. of Physiology and Biophysics, Bldg. RMSB Room 5089, University of Miami School of Medicine, 1600 NW 10 Ave, Miami FL 33136, USA; Telephone: 305-243-5963; Fax 305-243-3364; email: kmuller@miami.edu 
Comprehensive list of abbreviations:

aCSF, artificial cerebrospinal fluid

CCD, charge coupled device

C3-C5, third to fifth cervical

DMSO, dimethyl sulfoxide

E, embryonic day

SEM, standard error of the mean 


\section{ABSTRACT}

Unfailing respiration depends on neural mechanisms already present in mammals before birth. Experiments were made to determine how inspiratory and expiratory neurons are grouped in the brainstem of foetal mice. A further aim was to assess whether rhythmicity arises from a single pacemaker or is generated by multiple sites in the brainstem. To measure neuronal firing, a fluorescent calcium indicator dye was applied to embryonic central nervous systems isolated from mice. While respiratory commands were monitored electrically from C3-C5 ventral roots, activity was measured optically over areas containing groups of respiratory neurones, or single neurones, along the medulla from the facial nucleus to the pre-Bötzinger complex. Large optical signals allowed recordings to be made during individual respiratory cycles. Inspiratory and expiratory neurones were intermingled. A novel finding was that bursts of activity arose in a discrete area intermittently, occurring during some breaths, but failing in others. Raised $\mathrm{pCO}_{2}$ or lowered $\mathrm{pH}$ increased the frequency of respiration; neurons then fired reliably with every cycle. Movies of activity revealed patterns of activation of inspiratory and expiratory neurones during successive respiratory cycles; there was no evidence for waves spreading systematically from region to region. Our results suggest that firing of neurons in immature respiratory circuits is a stochastic process, and that the rhythm does not depend on a single pacemaker. Respiratory circuits in foetal mouse brainstem appear to possess a high safety factor for generating rhythmicity, which may or may not persist as development proceeds.

Key words: respiration; rhythm; calcium imaging; prenatal 
It is possible to commit suicide by not eating, but one cannot terminate the relentless rhythm of respiration by willpower. The rhythm is generated by brainstem neurons that activate motoneurones; these, in turn, give rise to contractions of inspiratory and expiratory muscles (Feldman et al., 2003). The activity of brainstem respiratory neurones is directly modified by altered levels of $\mathrm{CO}_{2}$ and $\mathrm{H}^{+}$, and indirectly by peripheral chemoreceptors (Richter and Spyer, 2001; Feldman et al., 2003; Hilaire and Pasaro, 2003). Whereas the circuitry that produces rhythmicity in postnatal mammals has been studied extensively (Bianchi et al., 1995; Ezure, 2004; Feldman et al., 2003; Ramirez and Richter, 1996), less is known about the organization of the respiratory network during embryonic life.

In preparation for birth, breathing movements, without air intake, occur in embryos (Boddy and Dawes, 1975). In mouse and chick, rhythmic activity appears when the rhombencephalon and respiratory circuits become defined through activation of homeotic genes (Champagnat and Fortin, 1997). By E16, fictive respiration of mice can be monitored from phrenic motoneurones (Viemari et al., 2003) .

A key question is whether foetal rhythms are produced by a group of pacemaker cells, or by reciprocal connections, such as those commanding motor behaviours in vertebrates and invertebrates (Marder and Calabrese, 1996). A focussed pacemaker would be expected to initiate activity spreading from one region of the medulla (Rybak et al., 2003). The spatial distribution in foetal brainstem of inspiratory and expiratory neurons is unknown. Experimental difficulties in analyzing developing respiratory neurons arise from the intrauterine location of the foetus, the ventral 
position of the pattern generator in the medulla, and from the limited number of neurons from which electrical recordings can be made simultaneously.

In the present experiments we recorded signals from neurons in isolated brainstem-spinal cord preparations after application of a calcium-sensitive fluorescent dye. Regions across the medulla were surveyed and their activity measured. In foetal preparations, unlike slices, brainstem respiratory nuclei are still in their normal relationships with each other and with motoneurons (Suzue, 1984). The rhythm of fictive respiration increases in frequency and reliability from E16 to E18, when the rate in the isolated preparation approximates that of the newborn pup (Viemari et al., 2003). This rhythm persists for more than 24 hours in vitro, even though all peripheral tissues are removed. That the mouse embryonic brainstem is thin and relatively transparent is advantageous for recording optical signals. Patterns of activity over foetal rat and newborn rat and mouse medulla were studied by Onimaru and his colleagues by means of voltage sensitive dyes (Onimaru and Homma, 2005; Onimaru et al., 2004; Onimaru and Homma, 2003). In those experiments the signal to noise ratio required averaging of 20-50 respiratory cycles. Calcium sensitive dyes could in principle give better signal-to-noise ratios, since calcium signals mirror integrated bursts rather than single impulses, and such dyes have been used in foetal mouse brain slices (ThobyBrisson et al., 2005). Moreover, $\mathrm{Ca}^{2+}$ signals arise primarily from cell bodies and the axon stump rather than axons and dendrites, in which less $\mathrm{Ca}^{2+}$ accumulation occurs (Przywara et al., 1991; Ross, 1989). 


\section{Experimental Procedures}

Experiments were made according to the Institute for Laboratory Animal Research Guide for the Care and Use of Laboratory Animals and were approved by Institutional Animal Care and Use Committees at the Marine Biological Laboratory and the University of Miami. Methods for euthanasia were in agreement with the "Report of the AVMA Panel on Euthanasia (2000)".

Animals: 34 CF1 and CD1 mouse embryos aged 16 to 20 days were removed by Caesarian section from pregnant animals (Charles River Laboratories Inc., Wilmington, MA) under anaesthesia (ketamine/xylazine, $80 / 20 \mathrm{mg} \mathrm{Kg}^{-1}$ i.p.); mothers were killed by anaesthetic overdose. Embryos were placed in $4{ }^{\circ} \mathrm{C}$ artificial cerebrospinal fluid (aCSF) and killed instantaneously by removal of the heart. aCSF contained (mM): $\mathrm{NaCl}, 125 ; \mathrm{NaHCO}_{3}, 24 ; \mathrm{KCl}, 5 ; \mathrm{CaCl}_{2}, 1.0 ; \mathrm{MgSO}_{4}, 1.25 ; \mathrm{KH}_{2} \mathrm{PO}_{4}$, 1.25; glucose, 30; equilibrated with $\mathrm{CO}_{2}: \mathrm{O}_{2}=5 \%: 95 \%(\mathrm{pH}=7.4)$. The cerebrum was removed by ponto-mesencephalic transection.

Calcium indicator dye: Brainstem spinal cords were incubated in $0.5 \mathrm{ml}$ of gassed aCSF containing fluorescent high-affinity calcium-sensitive dye, Oregon Green 488 Bapta-1, AM (Molecular Probes, Eugene, OR) initially dissolved in $10 \mu \mathrm{l}$ of $20 \%$ Pluronic F-127 in DMSO (Molecular Probes, Eugene, OR). The dye in its AM form is transported into cells and enzymatically cleaved to render it impermeant. Concentrations of $300-500 \mu \mathrm{g} \mathrm{ml}^{-1}$ and incubation periods of $45-60$ minutes were optimal for staining without reduction in respiratory frequency. The lower affinity dyes Oregon Green 488 BAPTA -2, AM $\left(300 \mu \mathrm{g} \mathrm{ml}^{-1}\right)$ and Oregon Green 488 BAPTA -6, AM $\left(150-600 \mathrm{~g} \mathrm{~m} \mathrm{~m}^{-1}\right)$ (Molecular Probes, Eugene, OR) were less effective. 
Electrical recording: Preparations were pinned to Sylgard 184 resin (DowCorning, Midland, Ml) in a $1.0 \mathrm{ml}$ chamber, and superfused continuously with gassed $\operatorname{aCSF}\left(3\right.$ to $\left.4 \mathrm{ml} \cdot \mathrm{min}^{-1}\right)$ at $23-25^{\circ} \mathrm{C}$ (room temperature), or at $29.5^{\circ} \mathrm{C}$ (temperature controller, TC-344B, Warner Instruments Co.). At the higher temperature the frequency of fictive respiration increased by about $50 \%$. Electrical signals recorded with suction micropipettes from C3-C5 ventral roots, were amplified by a P15 Grass amplifier (Grass Instrument Co., Quincy, MA), filtered at $100-3000 \mathrm{~Hz}$., and integrated with a full-wave rectifier ( $\tau=100 \mathrm{~ms})$. Electrical signals and optical images were stored in a computer. Medullary unit recordings were made with glass microelectrodes filled with $0.2 \mathrm{M} \mathrm{NaCl}$, resistance 3-8 $\mathrm{M} \Omega$.

Optical recording: Changes in calcium fluorescence were detected by a CCD camera (NeuroCCD-SM 256; RedShirtlmaging, LLC, Fairfield, CT, USA) and an epifluorescence microscope (Nikon Eclipse EF600 or Zeiss WL) with heat filter, fluorescein filter set, and 40x, $0.75 \mathrm{NA}$ water immersion objective. The CCD camera had an imaging area of $256 \times 256$ pixels with a time resolution to $10 \mathrm{~ms}$ and image diameter of 400 to $500 \mu \mathrm{m}$, depending on the microscope. Fluorescence changes were expressed as percentages (change in fluorescence intensity compared to the resting light intensity image). Changes of less than $1 \%$ could be detected. At the illumination intensities used, about $3 \%$ of the dye bleached during a 20 sec recording, with no effect on the regularity of the signal or on recordings from the same region made minutes later. To test for reliability, records were made from an area and then from distant areas of medulla, returning 15-20 minutes later to the original spot, where the activity remained unchanged. The plane of focus was altered to observe signals arising 
25-50 $\mu \mathrm{m}$ below the surface; the pattern of responses over an area was similar to that recorded from the surface. To avoid movement artefacts, the flow was stopped during optical recordings.

Data analysis: Optical and electrical data were acquired, and analyzed using the NeuroPlex program (RedShirtlmaging). Acquisition trials constituted a series of frames at 20-40 ms intervals, over $20 \mathrm{~s}$ or $40 \mathrm{~s}$. Signals were processed with $2 \times 2-$ pixel binning to reduce noise, and digitally with high- and low-pass temporal filters; kernel averaging permitted display of spatially averaged signals from selected regions of adjustable size ranging from circular fields of $60 \mu \mathrm{m}$ diameter to areas corresponding to the diameter of individual neurons. Recordings such as those in Figures 1, 2 and 4 concern phase relations and magnitudes of signals: their shapes depend on filtering. Sequences of images were displayed as videos (attached as Supplementary Material) using standard compression protocols with NeuroPlex. 


\section{Results}

\section{Optically recorded respiratory rhythms.}

Figure $1 A$ shows optical signals recorded from the ventral surface of the rostral medulla, caudal to the facial nucleus level during fictive respiration (E17 preparation). The lower trace (red) shows the integrated electrical activity from ventral root C5; expiration occurred between the peaks. The frequency of fictive respiration in 34 preparations was $15.5 \pm 1.0 \mathrm{~min}^{-1}$ (mean \pm SEM) and the duration of the inspiratory burst was $0.40 \pm 0.05 \mathrm{~s}$. Respiratory rhythms persisted for more than $7 \mathrm{~h}$ in preparations stained with dye, and unlike locomotor activity, were synchronous on both sides of the cervical spinal cord segment. The optical signals shown in Fig. $1 \mathrm{~A}$ originated in a $0.4 \mathrm{~mm}$ diameter region of medulla indicated by the larger circle in the upper-right rectangle. Twenty coloured circles represent selected areas over which optical signals were measured. Coloured traces show changes in fluorescence that accompanied respiration and averaged over corresponding areas. The optical signals in the areas marked red and pink, for example, were linked to inspiration and had excellent signal to noise ratios. For clarity, optical signals from only a few areas are shown in Fig. $1 A$, although the entire field in view with the 40x lens could be explored in detail and compared with displays of the data as video movies (see Fig. 2 and supplementary material). Features of optical signals were good signal to noise ratios for a single cycle, and widespread areas from which signals arose. When fields of activity were examined pixel by pixel, records like those of Fig. $1 B$ were obtained. Here the three areas surveyed (indicated by turquoise, brown and blue spots and traces), were at 6 pixels in diameter as small as the expected diameter of single neurons (two 
pixels corresponded to $\sim 3 \mu \mathrm{m}$, while the diameters of cell bodies ranged from 8 to $14 \mu \mathrm{m}$ ). When the spot was moved minimally (two or three pixels) from the central point, the signal became smaller or disappeared. Figure $1 B$ further shows expiratory (brown and blue traces), together with inspiratory activity (turquoise). Activity corresponding to expiration was observed rarely, compared to signals accompanying early phases of inspiration (in 10 areas out of 120 sampled showing phase locked signals). Occasionally, optical signals were not related to the phases of respiration. The distribution of brainstem areas showing respiratory activity was mapped by systematic exploration in 10 preparations. Optical signals arose over areas situated laterally along the medulla, from the facial nucleus level to the pre-Bötzinger complex level.

The spatial pattern of activity from breath to breath is shown over one field in Fig. 2 while the shutter was open for $40 \mathrm{~s}$ (Supplementary video). In this field with predominantly inspiratory activity frames $2 A$ and $C$ (timing indicated by thin black lines to integrated C5 phrenic nerve activity) display surges (red) in activity during inspiration, with lowered activity in Frames $2 B$ and $D$ related to expiration. If the rhythm of respiration in foetal midbrain had arisen from a single pacemaker, one might expect activity to spread from one region to another, say from rostral to caudal or vice versa. Inspection of continuous films of activity such as those used in Fig. 2 failed to reveal progressive waves across the fields, either systematically or in a different pattern with each breath.

\section{Intermittent signals at physiological pH.}

At $\mathrm{pH} 7.4$, patterns of optical activity recorded from a particular area were either regular (Fig. 1B), or intermittent. For example in Figure $1 A$ the adjacent areas coloured 
in red and pink gave large signals that failed in some cycles. In records from 3 experiments with good signal to noise ratios, 93 failures occurred in 309 respiratory cycles, while in adjacent areas failures did not occur or were out of phase. These results suggested that the recruitment of respiratory units might be stochastic during each cycle: all inspiratory neurons might not be used for every cycle.

Published electrical recordings made from single neurons in the medulla do not generally show intermittent failures (Ezure, 2004). Electrical recordings were made from respiratory neurons in foetal medulla to measure regularity. Fig. 3 shows burst durations of medullary units that vary with each cycle, with occasional failures. Apparent discrepancies between electrical and optical recordings might occur because calcium signals reflect bursts rather than single action potentials, and possibly because units that fire intermittently might have been ignored in earlier published experiments.

\section{Effects of changing $\mathrm{pH}$ or $\mathrm{pCO}_{2}$.}

Further evidence that optical signals corresponded to fictive respiration was provided by applying low $\mathrm{pH}$ or high $\mathrm{pCO}_{2}$ solutions to 15 isolated CNS preparations. Changes of $\mathrm{pH}$ from $\mathrm{pH} 7.3$ to 7.1 or 7.4 to 7.2 (brought about by increased $\mathrm{CO}_{2}$ or by lowered bicarbonate concentration), increased the respiration frequency by $52.9 \% \pm$ $11.4 \%(p=0.0015$, Wilcoxon signed-rank test, $n=15)$. Fig. 4 shows that failures of optical signals did not occur at $\mathrm{pH}$ values of 7.3 or lower. 


\section{Discussion}

\section{Calcium signals during respiratory activity.}

Changes in intracellular calcium concentration produced optical signals that were measurable during a single cycle without averaging, even when small areas were monitored. Presumably the excellent signal to noise ratio was possible, in part, because signals represented bursts rather than single action potentials, in cell bodies and dendrites. In studies made with voltage sensitive dyes, signals became large enough to analyse only after averaging the effects of 20 to 50 respiratory cycles (Onimaru and Homma, 2003; Onimaru et al., 2004; Onimaru and Homma, 2005). What cannot be said with certainty is the cellular basis for changes in optical activity. Although identified glial cells are not evident at early stages of development, their participation cannot be ruled out. The close correspondence between electrical records of medullary units and optical records suggests that neurones are responsible; to prove their role requires simultaneous electrical and optical recording from single neurons or glia, a formidable task. A further problem concerns the depths of cells that gave rise to optical signals. A limitation of the present technique is that successful signals cannot be obtained from deeper than a few hundred microns within the medulla.

\section{"Respiration" in foetal mice.}

The brainstem machinery for producing rhythmical movements for respiration is already present in mouse or human embryos. Prematurely born mammals can breathe the instant they are born, even though respiratory movements in the uterus do not entail intake of air (Viemari et al., 2003). 


\section{Intermittent failures of optical and electrical signals.}

Our finding that groups of medullary neurons associated with inspiration might not fire with every breath was unexpected. Published electrical recordings do not show "failures". What medullary units show is inconstancy of burst duration and frequency from breath to breath in newborn opossum (Zou, 1994) or foetal mouse (see for example Figure 3); such irregularity would be reflected as irregular calcium signals. The presence of "failures" suggests that the rhythm could be generated by a stochastic process of the type seen in other rhythm generators (Kopell and LeMasson, 1994). For example, in locomotor circuits not every neuron is active with every movement (Butt et al., 2002).

\section{Organisation of respiratory neurons in foetal mouse.}

Changes that occur in respiratory mechanisms as the foetus develops and becomes adult have been studied in several mammals (Hilaire and Duron, 1999), with emphasis on regulatory mechanisms, and the possible role of pacemaker neurons at successive stages (Del Negro et al., 2005; Peña et al., 2004). Maps of respiratory activity in newborn rat and mouse made with voltage sensitive dyes suggested that the respiratory activity started in each cycle on a discrete group of neurons located in the para-facial region, and then it was propagated caudally into the pre-Bötzinger complex level (Onimaru and Homma, 2003; Onimaru et al., 2004). By contrast, in animals after birth, the origin of the rhythm appeared to be related to the pre-Bötzinger complex (Feldman et al., 2003; Onimaru and Homma, 2005). In our experiments, the area examined in each recording field was smaller than that observed by Onimaru and his colleagues (Onimaru and Homma, 2003; Onimaru et al., 2004; Onimaru and Homma, 
2005). The area over which rhythmicity arose was assessed by moving from place to place on the ventro-lateral surface of the medulla, from the facial nucleus to the preBötzinger complex. From video records of activity over periods of $20-40 \mathrm{~s}$, we saw no evidence for a discrete group of pacemaker cells from which activity spread. In a particular region, activity did not proceed as a coordinated progressive wave. Instead, signals arose from the whole area in a synchronous, coordinated manner during inspiration. Moreover, the signal to noise ratio was good enough for each breathing cycle to be examined on its own without averaging. Our results suggest that, rather than a pacemaker generating the rhythm at one spot, whole areas could be active at once, as if rhythmicity depended on a diffuse network. Support for such an idea comes from records of fictive respiration in the newborn opossum (Eugenin and Nicholls, 1997). There it was found that application of fluid with altered $\mathrm{pH}$ from a micropipette to a localised area of medulla modified the rate of respiration. Similarly, localised application of procaine temporarily blocked respiratory activity (Eugenin and Nicholls, 2000). An important feature of these experiments was that results were obtained when the solution was applied locally to any point within the region containing respiratory units.

\section{Conclusion.}

Our results suggest that the circuitry in foetal brainstem that gives rise to the rhythm of respiration consists of a distributed neuronal network. Moreover, although neurones may not fire with every breath, they can all be recruited to fire unfailingly in low $\mathrm{pH}$ or high $\mathrm{CO}_{2}$. This type of circuit would provide a high safety factor for 
maintaining foetal respiration. It remains open whether similar mechanisms for generating the rhythm are present in the adult.

\section{Acknowledgements}

Supported by grants from FONDECYT \#1010242 and \#7010242 to JE, NIH DC05259 to LC, the State of FL to KM, and an Evelyn and Melvin Spiegel Foundation Award for a summer research fellowship at the MBL to JE. We thank Conrado Freites for apparatus construction and Dr. Chun Falk for guidance with NeuroPlex software and use of the NeuroCCD-SM 256 camera. 


\section{Figure Legends}

Figure 1. a. Fluorescence changes in rostral medulla (traces on the left) and integrated C4 "phrenic" ventral root burst activity (bottom trace) in brainstem-spinal cord preparation (E17 mouse, $5 \% \mathrm{CO}_{2}, \mathrm{pH} 7.4$ ), stained with calcium-sensitive dye. Scheme and photograph of the preparation (right) indicate, in colours corresponding to optical traces, the areas for averaging the fluorescence (right, top). Blue, red and pink lines, for example, join medullary areas surveyed for averaging with corresponding optical traces. Note that spots showing respiratory-related activity are distributed along a rostrocaudal column. b. Inspiratory- (top trace) and expiratory-related (second and third traces) fluorescence changes recorded optically from rostral medulla (E20 brainstem-spinal cord). Integrated C4 ventral root activity is shown below. Fluorescence signals were averaged over areas corresponding to cell bodies of fluorescent neurones. Colored lines join areas in medulla used with their respective optical recordings.

Figure 2. Pseudocolor images of fluorescence during inspiratory $(A, C)$ and expiratory $(\mathrm{B}, \mathrm{D})$ respiratory cycles. (E18 mouse brainstem preparation, $5 \% \mathrm{CO}_{2}, \mathrm{pH}$ 7.3.) Timing of optical recording frames $A-D$ is indicated by lines projecting to the integrated $C 5$ motoneuron activity. Within the field of view, the region of highest optical activity (red) corresponding to peak $\mathrm{Ca}^{2+}$ concentration is similar from one episode to the next during inspiration. In this region of strong optical activity during inspiratory phases, expiratory phases of activity are less evident. The full sequence of images is presented as a 
movie in a Supplementary Figure, which shows no progressive waves, but rather periodic surges of activity predominantly in the middle and right of the field.

Figure 3. Medullary units (Med unit), with unitary (C5) and integrated (Int) respiratory activity recorded from C5 ventral root. Burst durations and frequency of medullary units varied greatly, with what appeared to be failures in some cycles, as shown in the lower expanded traces. (E18 mouse embryo, $\mathrm{pH} 7.3,5 \% \mathrm{CO}_{2}$.)

Figure 4. Effect of acidification on respiratory rhythm in a preparation from an E18 mouse. The lower trace of integrated activity from $C 4$ (red) and optical activity showed increases in frequency from $12 \mathrm{~min}^{-1}$ to $18 \mathrm{~min}^{-1}$ when $\mathrm{pH}$ was changed from $\mathrm{pH} 7.3$ to 7.2 by raising $\mathrm{CO}_{2}$ from $5 \%$ to $10 \%$.

\section{Supplementary material.}

Videos taken from the experiment of Figure 2 ( E18 mouse brainstem) by combining frames taken every $40 \mathrm{~ms}$. Pseudo colour shows increased calcium concentration (red) in the spectrum. Below is integrated motoneuron activity recorded from C5 (as in Fig. 2). A small red spot moving on the electrical recording trace indicates the timing of frames. Videos 1 and 2 are processed to show activity in two over the surface of the medulla and in three dimensions, the third dimension indicating scaled change in fluorescence intensity, with red (up) most intense. During inspiration a peak of activity covers the entire field with no discernible movement in a particular direction. During expiration calcium signals are diffuse and noisy. 


\section{REFERENCES}

Bianchi AL, Denavit-Saubie M, Champagnat J (1995) Central control of breathing in mammals: neuronal circuitry, membrane properties, and neurotransmitters. Physiol $\operatorname{Rev} 75: 1-45$.

Boddy K, Dawes GS (1975) Fetal breathing. Br Med Bull 31:3-7.

Butt SJB, Lebret JM, Kiehn O (2002) Organization of left-right coordination in the mammalian locomotor network. Brain Res Brain Res Rev 40:107-117.

Champagnat J, Fortin G (1997) Primordial respiratory-like rhythm generation in the vertebrate embryo. Trends Neurosci 20:119-124.

Del Negro CA, Morgado-Valle C, Hayes JA, Mackay DD, Pace RW, Crowder EA, Feldman JL (2005) Sodium and calcium current-mediated pacemaker neurons and respiratory rhythm generation. J Neurosci 25:446-453.

Eugenin J, Nicholls JG (1997) Chemosensory and cholinergic stimulation of fictive respiration in isolated CNS of neonatal opossum. J Physiol (Lond) 501 ( Pt 2):425437.

Eugenin J, Nicholls JG (2000) Control of respiration in the isolated central nervous system of the neonatal opossum, Monodelphis domestica. Brain Res Bull 53:605613.

Ezure K (2004) Respiration-related afferents to parabrachial pontine regions. Respir Physiol Neurobiol 143:167-175. 
Feldman JL, Mitchell GS, Nattie EE (2003) Breathing: rhythmicity, plasticity, chemosensitivity. Ann Rev Neurosci 26:239-266.

Hilaire G, Duron B (1999) Maturation of the mammalian respiratory system. Physiol Rev 79:325-360.

Hilaire G, Pasaro R (2003) Genesis and control of the respiratory rhythm in adult mammals. News Physiol Sci 18:23-28.

Kopell N, LeMasson G (1994) Rhythmogenesis, amplitude modulation, and multiplexing in a cortical architecture. Proc Natl Acad Sci U S A 91:10586-10590.

Marder E, Calabrese RL (1996) Principles of rhythmic motor pattern generation. Physiol Rev 76:687-717.

Onimaru H, Arata A, Arata S, Shirasawa S, Cleary ML (2004) In vitro visualization of respiratory neuron activity in the newborn mouse ventral medulla. Brain Res Dev Brain Res 153:275-279.

Onimaru H, Homma I (2003) A novel functional neuron group for respiratory rhythm generation in the ventral medulla. J Neurosci 23:1478-1486.

Onimaru H, Homma I (2005) Developmental changes in the spatio-temporal pattern of respiratory neuron activity in the medulla of late fetal rat. Neurosci 131:969-977.

Peña F, Parkis MA, Tryba AK, Ramirez JM (2004) Differential contribution of pacemaker properties to the generation of respiratory rhythms during normoxia and hypoxia. Neuron 43:105-117. 
Przywara DA, Bhave SV, Bhave A, Wakade TD, Wakade AR (1991) Stimulated rise in neuronal calcium is faster and greater in the nucleus than the cytosol. FASEB $J$ $5: 217-222$

Ramirez JM, Richter DW (1996) The neuronal mechanisms of respiratory rhythm generation. Curr Opin Neurobiol 6:817-825.

Richter DW, Spyer KM (2001) Studying rhythmogenesis of breathing: comparison of in vivo and in vitro models. Trends Neurosci 24:464-472.

Ross WN (1989) Changes in intracellular calcium during neuron activity. Ann Rev Physiol 51:491-506.

Rybak IA, Shevtsova NA, St John WM, Paton JF, Pierrefiche O (2003) Endogenous rhythm generation in the pre-Botzinger complex and ionic currents: modelling and in vitro studies. Eur J Neurosci 18:239-257.

Suzue T (1984) Respiratory rhythm generation in the in vitro brain stem-spinal cord preparation of the neonatal rat. J Physiol (Lond) 354:173-183.

Thoby-Brisson, M., Trinh, J.-B., Champagnat, J., and Fortin, G. (2005) Emergence of the pre-Bötzinger respiratory rhythm generator in the mouse embryo. 25: 43074318 Journal of Neuroscience.

Viemari JC, Burnet H, Bevengut M, Hilaire G (2003) Perinatal maturation of the mouse respiratory rhythm-generator: in vivo and in vitro studies. Eur J Neurosci 17:12331244. 
Eugenin et al.

Zou DJ (1994) Respiratory rhythm in the isolated central nervous system of newborn opossum. J Exp Biol 197:201-213. 
Figure 1
Click here to download high resolution image

A

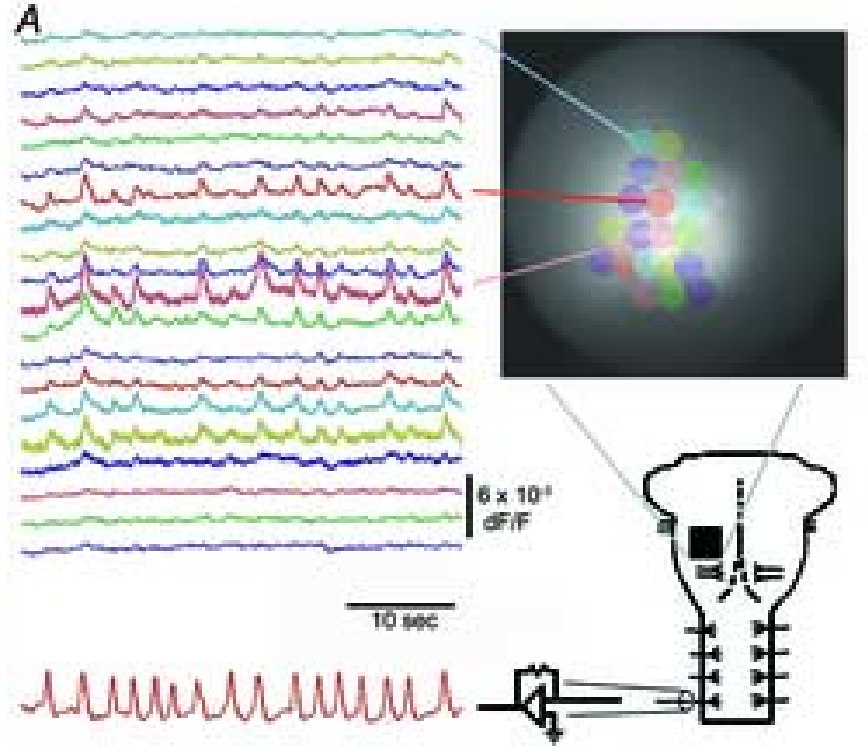

B

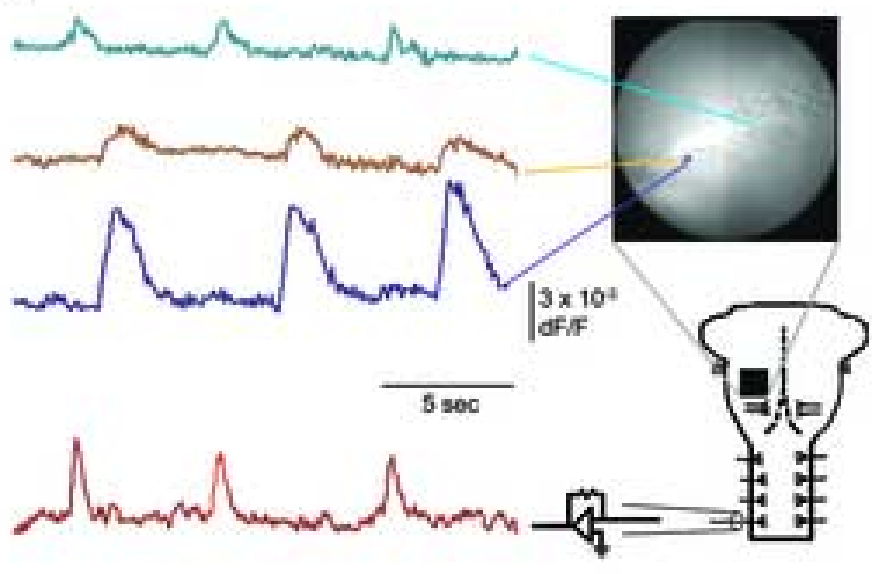

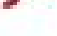

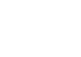

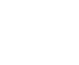

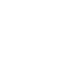

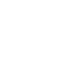

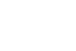
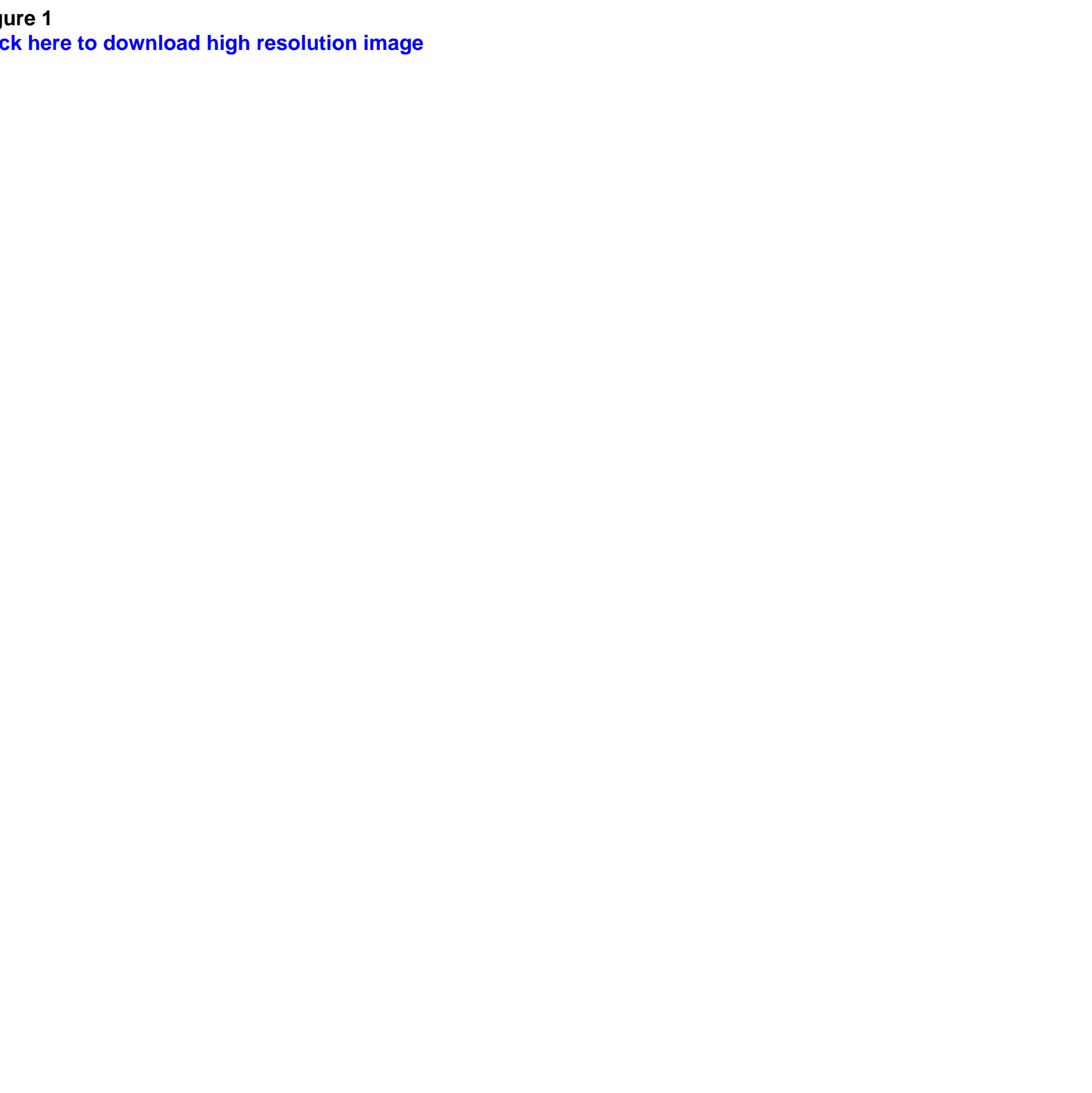
Figure 2
Click here to download high resolution image
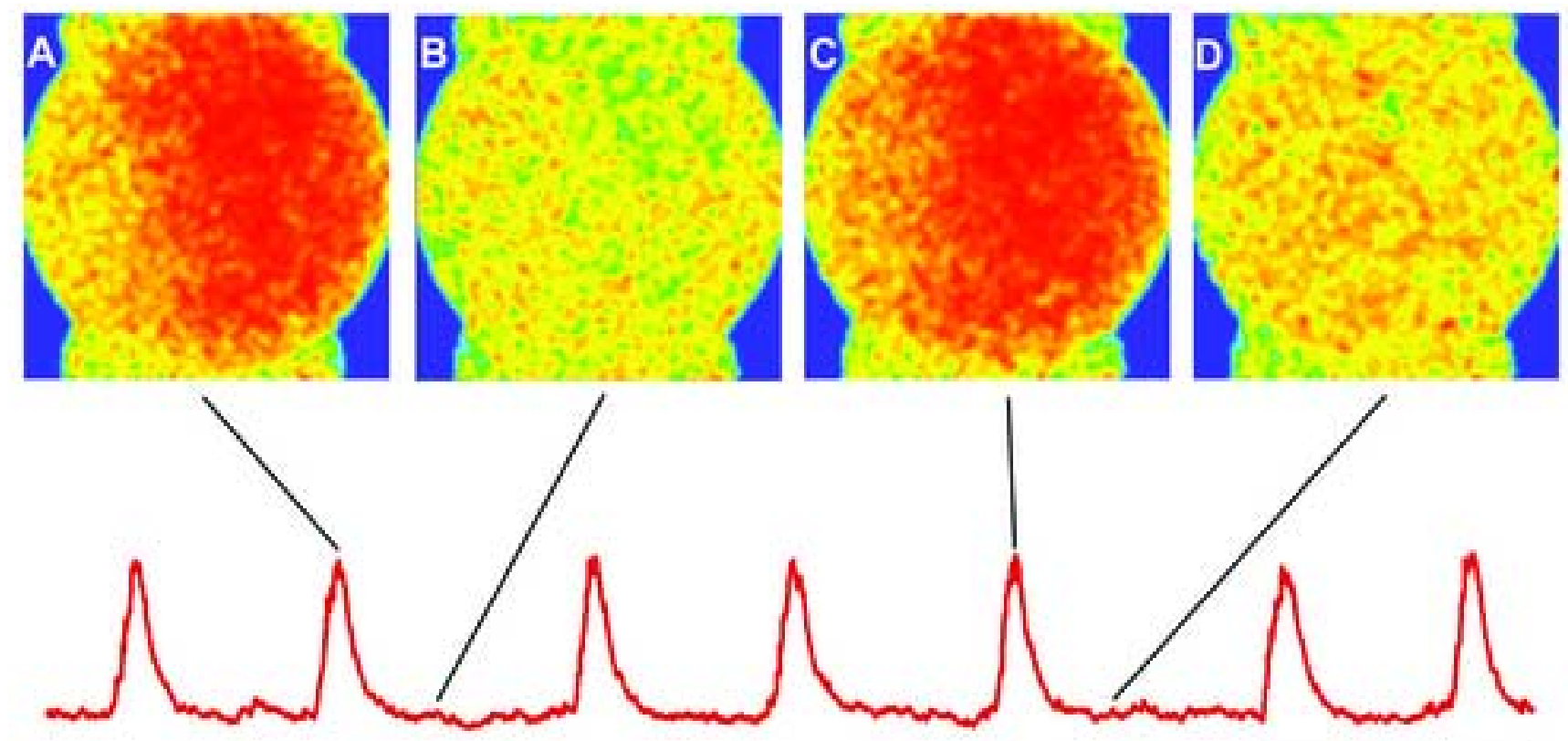

Int C5

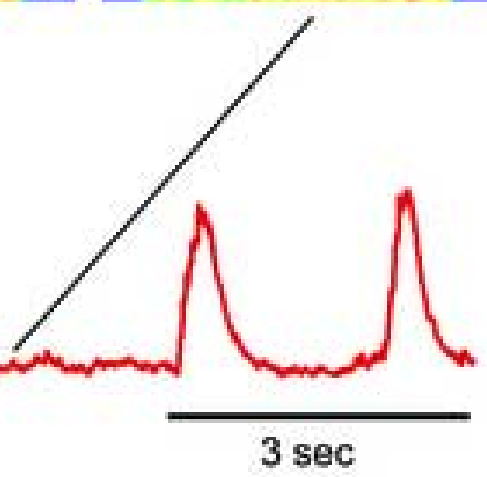


Click here to download high resolution image

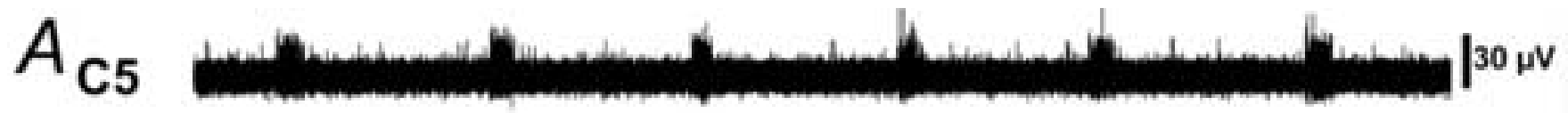
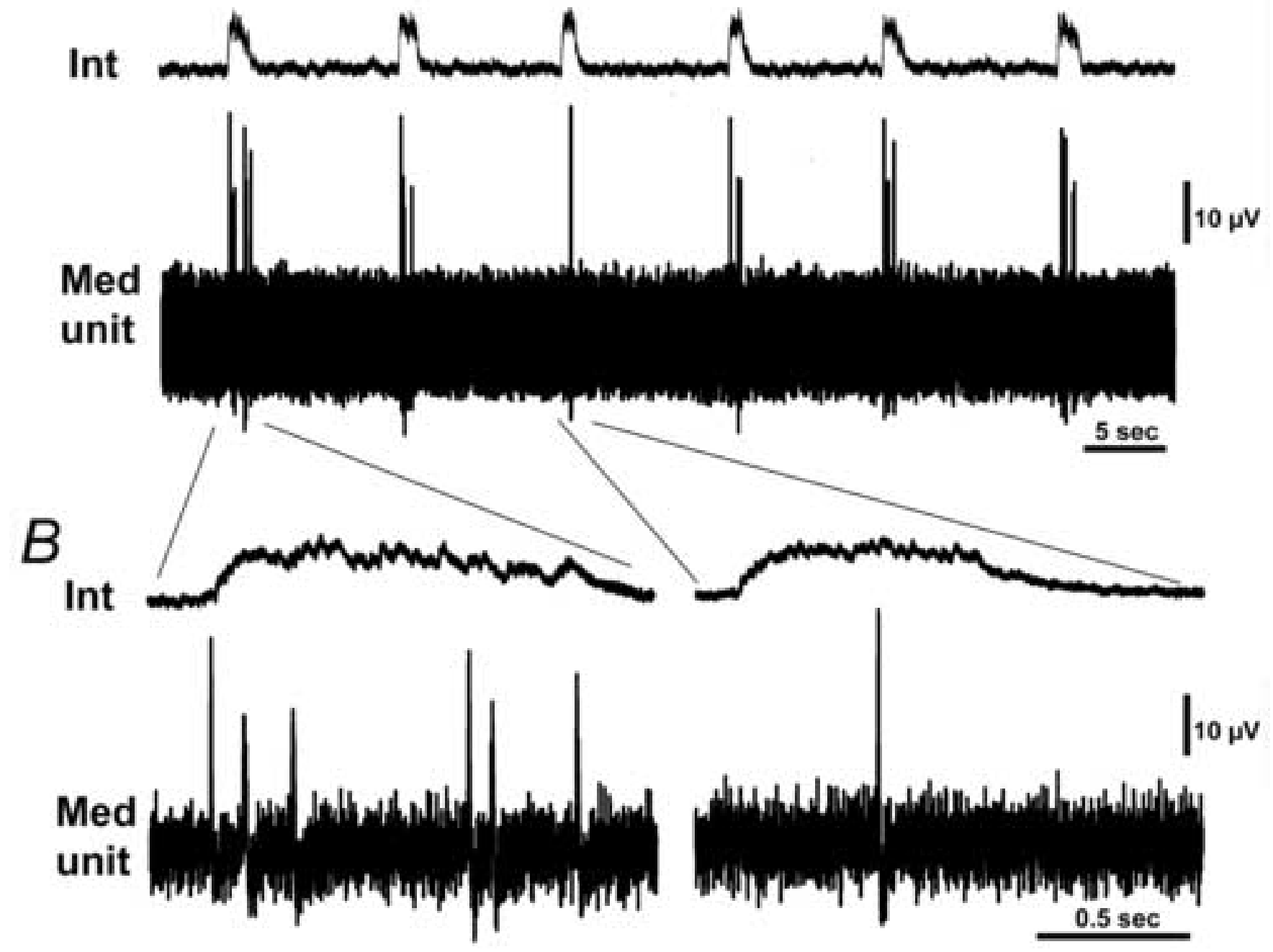
Supplementary Material--Video 1
Click here to download Supplementary Material: Eugenin Sup 1.mpg

Supplementary Material--Video 1
Click here to download Supplementary Material: Eugenin Sup 1.mpg 
Supplementary Material--Video 2
Click here to download Supplemer

Click here to download Supplementary Material: Eugenin Sup 2.mpg

\section{g

